\title{
Gyrotron frequency ECRIS development and the future challenges
}

\author{
L. Sun, H.W. Zhao, J.W. Guo \\ Institute of Modern Physics, CAS, Lanzhou 730000, China, sunlt@impcas.ac.cn
}

Gyrotron frequency amplifier has been used for the production of highly charged ion beams with high performance ECR ion sources (ECRIS) for almost 20 years. The widely used technology of microwave power coupling to the high temperature ECR plasma is still a borrowed design from the fusion machines. ECR ion source technologies and physics are advancing over the years, but the interpretation of how gyrotron frequency microwave interacts with ECR plasma and how to improve the ECRH efficiency still got little progress. Recently, experimental tests of $24 \mathrm{GHz}$ with different microwave modes and waveguide openings have succeeded at IMP with some very encouraging but surprising results. This paper will discuss the international needs of gyrotron amplifiers in the ECRIS community and the highlights of $\geq 24 \mathrm{GHz}$ microwave coupling. Typical questions and challenges in future development will be presented, especially how to meet the high performance needs of a 4 th generation ECR ion source.

ECRIS concept was proposed by Geller in late 1960s [1]. ECRIS was actually developed from plasma fusion device. Plasma electrons are heated through ECR heating to high energy by coupled microwave power with the frequency in the range of $2.45 \mathrm{GHz}$ to $28 \mathrm{GHz}$. The plasma is confined by a strong nested so-called mini-B magnetic field configuration (Fig. 1), which is the superposition of an axial mirror field and a radial multiple field (normally a hexapole field). Hot electrons are confined at the center of the nested field and the HCIs, which are produced by stepwise ionization while they are staying in the plasma, are trapped by the space charge established by the electrons inside the plasma. The lost ions that enter the extraction region will be accelerated by the applied $\mathrm{HV}$ potential to form intense mixed ion beams. HCI beams can be selected from the mixing beams with an analyzer magnet.

There are variable choices of HCI sources for heavy ion accelerators such as ECRIS, EBIS, MEVVA and LIS, and so on, however ECRIS remains an indispensable machine for worldwide heavy ion accelerator development. For high charge state intense $\mathrm{CW}$ or long pulse $(\sim \mathrm{ms})$ ion beams production, ECR in source is the most powerful tool. In recent 15 years, ECRIS performance has been boosted enormously, for instance $\mathrm{CW} \mathrm{X \textrm {Xe } ^ { 3 0 + }}$ beam was only 10-15 e $\mu \mathrm{A}$ from a state-of-the-art ECRIS about 14 years ago, and this number has been multiplied by a factor of at least 20 with one of the $3^{\text {rd }}$ generation ECRISs SECRAL [2]. The tremendous improvement is driven by the strong needs from modern advanced heavy ion accelerator projects or facilities, such as FRIB, SPIRAL2, FAIR, HIRFL/IMP, RIBF/RIKEN and so on. The application of gyrotron frequency microwave ECRH in the production of HCI beams plays significant role. A 28 $\mathrm{GHz}$ microwave amplifier was firstly tried in INFN/Catania with a superconducting ECRIS named
SERSE [3]. Borrowed microwave coupling technique from fusion machine was used in the test. Despite the insufficient magnetic confinement to ECR plasma, the 28 $\mathrm{GHz}$ ECRH gave many very promising beam intensity records around 2000. The first optimum ECRIS working at gyrotron frequency is VENUS which was test at 28 $\mathrm{GHz}$ microwave power in 2004 [4], and therefore the ECRIS community stepped into the real $3^{\text {rd }} \mathrm{G}$. era.

As a conventional technique, the existing $3^{\text {rd }} \mathrm{G}$. ECRISs are all using $\mathrm{TE}_{01}$ mode travelling in an oversized $\varnothing 32 \mathrm{~mm}$ opening circular waveguide as the gyrotron microwave power coupling scheme, which is actually a directly borrowed technique from fusion community. Ion sources working at gyrotron frequency have been tested and the capacity to produce more intense highly charged ion beams with sufficient magnetic confinement has been verified as had been predicted by the frequency scaling laws. However, at the same microwave power level or same power density level, gyrotron frequency heating is not doing as well as predicted. In most occasions, compared to $18 \mathrm{GHz}$, it is behaving just like a linear extrapolation of power effect to get more intense highly charged ion beams. At $24 \mathrm{GHz}$, SECRAL can produce the beam intensity of the $\omega^{2}$ scaling, but at much higher microwave power level compared to that at 18 GHz. Similar results have also been observed with SuSI [5]. This raised the question about the coupling efficiency of gyrotron microwave power into ECRIS plasma. Therefore, studies concerning gyrotron microwave coupling and ECRH have been done in both LBNL and IMP. Different microwave injection schemes have been tried with SECRAL at $24 \mathrm{GHz}$, such as $\mathrm{HE}_{11}$ mode, $\mathrm{TE}_{11}$ mode, or $\mathrm{TE}_{01}$ mode with variable waveguide openings. When an Ø20 mm TE $\mathrm{TE}_{01}$ mode oversized waveguide was installed, a significant different microwave coupling behavior was observed compared to other cases. As shown in Fig. 2, the output of the ion source responded to the incident 24 $\mathrm{GHz}$ microwave very linearly. No saturation was found even at the power limit of the $24 \mathrm{GHz}$ amplifier, i.e. 7.0 $\mathrm{kW}$, which was corresponding to a power density of $\sim 1.6$ $\mathrm{kW} / 1$. What is more important is that the production efficiency at the same RF power level for the case of $\varnothing 20$ $\mathrm{mm} \mathrm{TE}_{01}$ mode microwave is at least $30 \%$ higher than that of the $\varnothing 32 \mathrm{~mm} \mathrm{TE} \mathrm{TE}_{01}$ mode or the $\varnothing 20 \mathrm{~mm} \mathrm{HE} \mathrm{H}_{11}$ mode. As is well known, for a high charge state ECR ion source, the plasma operates at a low plasma density, and therefore the plasma chamber can be regarded as a multimode cavity [1]. For a $3^{\text {rd }}$ G. ECRIS, the plasma chamber diameter is typically at the dimension of $\varnothing>100 \mathrm{~mm}$, which is much larger than that of the incident gyrotron frequency RF wavelength. For this reason, the ECRH may not be sensitive to the change of incident microwave modes. However, the microwave power coupling has consequences on how the microwave power is fed into the chamber, i.e. the microwave coupling to ECR plasma 
and the coupling efficiency in terms of high charge state ion beam production. The interpretation to these results still need more experimental investigations and theoretical analysis.

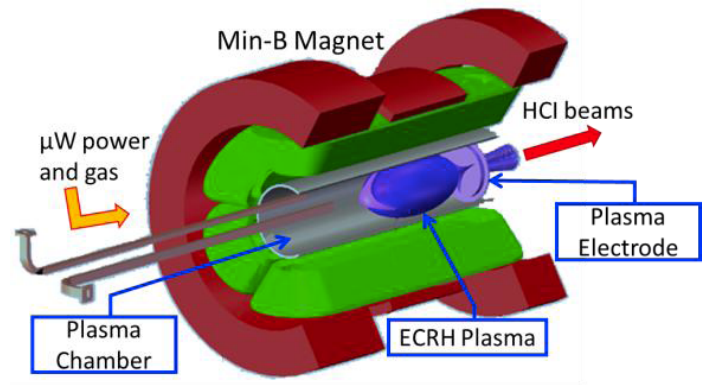

Fig. 1. Schematic plot of ECRIS

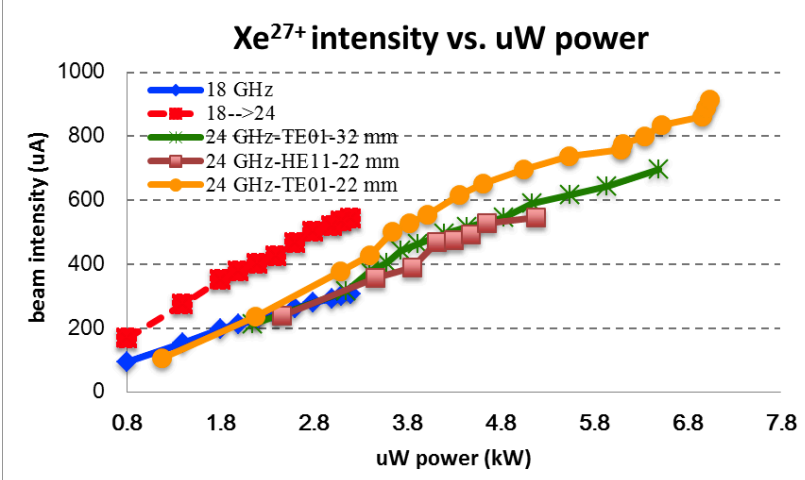

Fig. 2. ECRH effectiveness with different modes

HIAF or High Intensity heavy ion Accelerator Facility project to be launched in China, needs $50 \mathrm{p} \mu \mathrm{A}$ of $\mathrm{U}^{34+}$ from the injector ion source. The state of the art high performance ECR ion source such as VENUS can produce a beam intensity of $\sim 11.7 \mathrm{p} \mu \mathrm{A} \mathrm{U}^{34+}$ [6]which is barely $1 / 4$ of the desired beam intensity. Obviously, the $3^{\text {rd }} \mathrm{G}$. ECRISs still have margins for performance enhancement through technical improvements or new method applications, such as the ECRH scheme modification as presented in former paragraph. However, to have such a high gain factor, a more powerful machine is an inevitable approach. According to the history of ECRIS in the last four decades, the frequency scaling governs its development. Therefore, a $4^{\text {th }}$ G. ECRIS with a performance of 4 times higher than the $3^{\text {rd }} \mathrm{G}$. machines, is typically working at a frequency of 40 50 GHz. A 45 GHs ECRIS named FECR is under intense R\&D at IMP. There are many technical and physics challenges that need longterm R\&D and probably some big break-through in the development, such as $\mathrm{Nb}_{3} \mathrm{Sn}$ magnet construction, cryogenics solution, intense beam extraction and transmission, and so on [7]. Specifically, microwave power transmission, coupling and ECRH will be critical issues for FECR. Since the wavelength of $45 \mathrm{GHz}$ microwave is $\sim 6.67 \mathrm{~mm}$, quasi-optical transmission scheme is routinely utilized for high power transmission. This scheme has already been widely utilized in the fusion machines, but to accommodate with an ECRIS, the outcome is not evidenced yet. Many technical details and modifications will be made accordingly. As is demonstrated with $3^{\text {rd }} \mathrm{G}$. ECRISs, to achieve the performance predicated by scaling laws at 24 or $28 \mathrm{GHz}$, higher power density inside the plasma chamber is needed compared to that of $18 \mathrm{GHz}$, which leads to the typical problems such as chamber cooling, bremsstrahlung radiation, cryogenic heat load and so on. For a $45 \mathrm{GHz}$ ECRIS, we are inevitably encountering the same problems, or even worse. Therefore, a better understanding on how gyrotron frequency microwave heating works with a high performance ECRIS is urgent. FECR is designed to be operated at $45 \mathrm{GHz}$ with a maximum input microwave power of $20 \mathrm{~kW}$, i.e. a corresponding microwave power density of $\sim 2 \mathrm{~kW} / 1$, which is not so challengeable, but the localized plasma loss to the chamber wall will give a $>10 \mathrm{MW} / \mathrm{m}^{2}$ power flux dump that will destroy an Al chamber wall easily. Consequently, it has the highest priority to improve the ECRH effectiveness at gyrotron frequency. Probably, very high power injection might be avoided so as to mitigate the other problems.

This work is supported by NSF (contract No. 11427904), MOST (contract No. 2014CB845500) and NSF (contract No. 11375244). The fruitful discussion with C. M. Lyneis, D. Z. Xie from LBNL and T. Thuillier from LPSC/Grenoble is warmly acknowledged.

\section{References}

1. Geller R. Electron Cyclotron Resonance Ion Sources and ECR Plasmas// Institute of Physics Publishing Bristol and Philadelphia, 1996. P. 85-92.

2. Sun L., Guo J. W., Lu W., et al. Advancement of highly charged ion beam production by superconducting ECR ion source SECRAL (invited)// Rev. Sci. Instrum. 2016. 87. 02 A707.

3. Gammino S., Ciavola G., Celona L., Hitz D., Girard A., Melin $G$. Operation of the SERSE superconducting electron cyclotron resonance ion source at $28 \mathrm{GHz} / / \mathrm{Rev}$. Sci. Instrum. 2001. V. 72, No. 11. P.4090-4097.

4. Leitner D., Lyneis C. M., Abbott A. R., Dwinell R. D., Collines D., Leitner M. First Results of the Superconducting ECR Ion Source VENUS with $28 \mathrm{GHz} /$ AIP Conference Proceedings, 2005. V. 749. P. 3-9.

5. Machicoane M., Cole D., Holland K., Leitner D., Morris $D$., Neben D., Tobos L. First Results at $24 \mathrm{GHz}$ with the Superconducting Source for Ions (SuSI)// Proceedings of $21^{\text {st }}$ Int. Workshop on ECR Ion Sources, 2014, http://www.jacow.org. P. 1-4.

6. Benitez J., Franzen K. Y., Lyneis C., Phair L., Strohmeier M., Machicoane G., Sun L. T. Current Development of the VENUS Ion Source in Research and Operations// Proceedings of $20^{\text {th }}$ Int. Workshop on ECR Ion Sources, 2012, http://www.jacow.org. P.153-158.

7. Sun L., Zhao H. W., Guo J. W., Yang Y., Lu W., Wu W., Ma L. Z. Challenges for the Next Generation ECRIS // Proceedings of HIAT2015, 2015, http://www.jacow.org. P. 268-273. 